\title{
The Balmer extinction sequence in spiral galaxies
}

\author{
L. Sodré Jr. ${ }^{1}$, A. Mateus Jr. ${ }^{1}$, G. Stasińska ${ }^{2}$ and R. Szczerba ${ }^{3}$ \\ ${ }^{1}$ Departamento de Astronomia, Instituto de Astronomia, Geofísica e Ciências Atmosféricas da \\ USP, Rua do Matão 1226, 05508-090, São Paulo, Brazil \\ ${ }^{2}$ LUTH, Observatoire de Meudon, 5 Place Jules Janssen, F-92195 Meudon Cedex, France \\ ${ }^{3}$ N.Copernicus Astronomical Center, Rabiańska 8, 87-100 Toruń, Poland
}

\begin{abstract}
Using spectra of normal emission line galaxies from the First Data Release of the Sloan Digital Sky Survey (SDSS) we have investigated the relations between the extinction $C(\mathrm{H} \beta)$ as derived from the $\mathrm{H} \alpha / \mathrm{H} \beta$ emission line ratio and various global parameters of the galaxies. Our main findings include: $C(\mathrm{H} \beta)$ is linked with the galaxy spectral type and colour, decreasing from early- to late-type spirals. It also increases with increasing metallicity and is larger for more luminous galaxies and those with older stellar populations. The extinction of the stellar light is correlated with both the extinction of the nebular light and the intrinsic galaxy colours. The optical properties of the luminous infrared galaxies in our SDSS sample are very similar to those of our entire sample of SDSS galaxies. These results have implications for the determination of global star formation rates and total stellar masses in normal galaxies.
\end{abstract}

\section{Introduction}

Accounting for the presence of dust is paramount for our understanding of the constitution and evolution of galaxies. Indeed, dust modifies the light we receive from galaxies by both dimming it and modifying its colour. The determination of the bolometric luminosity of galaxies, the description of their stellar populations using galaxy colours, the estimates of the star formation rates using observed fluxes in either the emission lines or ultraviolet continua, all depend on a correction for the effects of dust.

Here we make use of observations from the First Data Release (DR1; Abazajian et al. 2003, see also Stoughton et al. 2002) of the SDSS to study the relation between the Balmer extinction $C(\mathrm{H} \beta)$ (i.e. the extinction at the wavelength of $\mathrm{H} \beta$, derived from the $\mathrm{H} \alpha / \mathrm{H} \beta$ ) and other global properties of the galaxies. We have selected 9840 galaxies, for which we measured equivalent widths and line ratios for the relevant spectral features. The Balmer extinction was then determined and correlated with other global properties of the galaxies, like their luminosities and nebular metallicities. More details may be found in Stasińska et al. (2004).

\section{Results}

Our main findings are the following: (a) $C(\mathrm{H} \beta)$ is linked with the galaxy spectral type and colour, decreasing from early- to late-type spirals. (b) $C(\mathrm{H} \beta)$ increases with increasing metallicity. (c) $C(\mathrm{H} \beta)$ is, probably, also affected by the age of the stellar population, being larger in the case of older stellar populations. (d) $C(\mathrm{H} \beta)$ depends on galaxy masses. (e) The extinction of the stellar light is correlated with both the extinction of the nebular light and the intrinsic galaxy colours, resulting in a trend with galaxy colour that may be opposite to the trend of $C(\mathrm{H} \beta)$. 
The fact that $C(\mathrm{H} \beta)$ correlates so well with other properties of galaxies is remarkable, given that the extinction, especially in late-types, is known to be not uniform across the face of galaxies (e.g. Beckman et al. 1996).

We have cross-correlated our sample of SDSS galaxies with the IRAS database in order to investigate any relationship between $C(\mathrm{H} \beta)$ and total infrared luminosity of the galaxies. Due to the lower redshift limit imposed to our sample and to the detection limit of IRAS, such a procedure selected only luminous infrared galaxies. We found that correlations that were shown by other authors to exist between optical and infrared properties of galaxies disappear when restricting to luminous infrared galaxies. We also found that the optical properties of the luminous infrared galaxies in our SDSS sample are very similar to those of our entire sample of SDSS galaxies.

We have proposed a phenomenological interpretation of our findings. We suggest that the main driver of the Balmer extinction of galaxies is their mass, combined with their metallicity and presence of old stellar populations. The infrared luminosity of the galaxies as determined by IRAS, which is attributed to radiation from hot stars reprocessed by dust grains, samples the regions with the most recent episodes of star formation, and is not connected with the Balmer extinction.

An important outcome of our study is to open the way for an improved correction for extinction in the determination of such parameters as the global star formation rate in galaxies or their total stellar masses. For normal galaxies, the global star formation rate can be obtained from the total $\mathrm{H} \alpha$ luminosity corrected for extinction using the extinction derived from the $\mathrm{H} \alpha / \mathrm{H} \beta$ emission line ratio (keeping in mind the reservations expressed e.g. by Hirashita et al. 2003). If observations do not allow one to determine the Balmer extinction, one can make use of e.g. the observed galaxy colour or the $D(4000)$ parameter to obtain an estimate of statistical value, since we have shown that all these quantities are correlated. On the contrary, the total stellar mass can be estimated from the observed stellar fluxes of the galaxy after correcting for stellar extinction. This should be done with a proper model fitting of the observed continuum as in Kauffmann et al. (2003). However, the strong correlation that we have found empirically between stellar extinction, Balmer extinction and galaxy colours can provide a basis for a statistical method to determine the total masses of galaxies. These aspects will be developed in future work and should be important especially for the study of galaxies at intermediate and high redshifts.

\section{Acknowledgements}

This work has benefited from grants from the PICS franco-brésilien, from the jumelage France-Pologne and from the Observatoire de Paris. A.M. and L.S. acknowledge support from FAPESP and CNPq; L.S is also grateful to CCINT/USP. The Instituto de Astronomia, Geofísica e Ciências Atmosféricas da USP has provided hospitality to G. S. and the Observatoire de Paris to L. S. and R. S.

\section{References}

Abazajian, K., et al. 2003, AJ, 126, 2081

Beckman, J. E., Peletier, R. F., Knapen, J. H., Corradi, R. L. M., \& Gentet, L. J. 1996, ApJ, 467,175

Hirashita, H., Buat, V., \& Inoue, A. K. 2003, ApJ, 410, 83

Stasińska, G., Mateus, A., Sodré, L., \& Szczerba. R. 2004, A\&A, in press (astroph/0403531)

Stoughton, C., et al. 2002, AJ, 123, 485 